Original article

\title{
Serum Vitamin D in Patients with Non Alcoholic Fatty Liver Disease
}

Mustafa s. Elkady ${ }^{\text {a }}$, Hatem S. Alegaily ${ }^{\text {a }}$, Hany R. ElKholy ${ }^{a}$, Shorouk Z. Abd-Elshafy ${ }^{\text {b }}$, Amal A. Qayed $^{\mathrm{c}}$

a Department of Hepatology, Gastroenterology and Infectious Diseases, Benha faculty of medicine, Benha University, Egypt. b Department of Diagnostic and Interventional Radiology, Faculty of Medicine, Benha University, Benha, Egypt. ${ }^{\mathrm{c}}$ Department of Hepatology, Gastroenterology and Infectious Diseases, Shebin El-Kom Teaching Hospital, Shebin El-Kom, Egypt.

Correspondence to: Amal A. Qayed, Department of Hepatology, Gastroenterology and Infectious Diseases, Shebin El-Kom Teaching Hospital, Shebin El-Kom, Egypt.

Email:

amalqayed@yahoo.com

Received:

Accepted:

\begin{abstract}
Background and study aim. Non alcoholic fatty liver disease has an increasing prevelance worldwide. It is closely associated with metabolic syndrome. The aim of this study is to assess the association between non alcoholic fatty liver disease and serum level of Vitamin D.
\end{abstract} Subjects and Methods. This cross sectional study was conducted on 95 subjects devided into 2 groups (1st group 45 patients with sonographically proven nonalcoholic fatty liver disease and 2nd group 50 healthy subjects). All the patients were evaluated by thorough full history taking, clinical examination, routine laboratory investigations, lipid profile, abdominal ultrasound and serum level of Vitamin D by ELISA. Results. Mean weight, Body mass index, and Waist circumference were significantly higher in patients with non alcoholic fatty liver disease than those without also they were significantly higher in patients with severe steatosis ( $\mathrm{P}$ value $0.001,0.000,0.002$ respectively). Incidence of Diabetes mellitus, Hypertension and Cardiovascular disease were significantly higher in patients with non alcoholic fatty liver disease than those without $(\mathrm{P}$ value $0.001,0.003$ and 0.009 respectively).Serum level of Vitamin D was significantly lower in non alcoholic fatty liver disease patients than those without also their values were lower in overweight NAFLD patients than those with normal body mass index but with no significant difference. Conclusion: Serum level of Vitamin D was highly deficient in patients with non alcoholic fatty liver disease than those without and its level significantly decreased with increasing grades of non alcoholic fatty liver disease.

Key words: Vitamin D, Fatty liver, NAFLD 


\section{Introduction:}

Nonalcoholic fatty liver disease (NAFLD) is defined as fatty infiltration of the liver proven by histology or radiology in the absence of secondary causes of steatosis such as alcoholic liver disease, drug-induced liver disease and viral hepatitis [1]. Features of metabolic syndrome like obesity, dyslipidemia, type 2 diabetes mellitus and hypertension are highly prevelant in patients with NAFLD and also increase risk of its developing [2]. Magnetic resonance imaging either by spectroscopy or by proton density fat fraction is an excellent noninvasive modality for quantifying hepatic steatosis and is widely used in NAFLD clinical trials [3].Ultrasound is still an important, cheap, and easy-to-use imaging tool for the diagnosis and grading of fatty liver diseases [4].

Liver biopsy is considered the gold standard for diagnosing steatosis but it is an invasive method assosciated with many adverse effects [5].Although NAFLD is the most common cause of hypertransaminasemia in children and adolescents, elevated Alanine aminotransferase (ALT) is not a sensitive marker of disease existence and/or severity at ordinarily used thresholds but the evaluation of both aspartate aminotransferase (AST) and ALT values is essential because an increased
AST/ALT ratio can reflect a progressive and more severe condition, such as fibrotic NASH [6].Vitamin D is capable of reducing free fatty acids induced insulin resistance in both peripheral tissues and hepatocytes, therefore low serum Vitamin D may predispose to intrahepatic lipid accumulation leading to NAFLD [7]. It is proved that serum levels of 25-hydroxyvitamin D are lower in subjects with NAFLD or NASH when compared with healthy controls so its insufficiency plays a role in the pathogenesis of NAFLD [8]. Vitamin D is capable of reducing free fatty acid (FFA) induced insulin resistance (IR) both in peripheral tissues and in hepatocytes so low serum Vitamin $\mathrm{D}$ is known to predispose to intrahepatic lipid accumulation leading to NAFLD [9]. It is proved that serum levels of 25-hydroxyvitamin D are lower in subjects with NAFLD or NASH when compared with healthy controls so its insufficiency plays a role in the pathogenesis of NAFLD [10]. Vitamin D has been implicated in immune modulation, cell differentiation and proliferation, and regulation of inflammation suggesting that Vitamin D may be a target for therapeutic intervention in the treatment of NAFLD and NASH [11]. Active Vitamin D is proved to modulate the immune system, 
induce an anti-inflammatory and antifibrogenic pattern in the liver and significantly inhibit the hepatic expression of pro-fibrotic mediators [12].Our aim is to study the assosciation between Non alcoholic fatty liver disease and Serum Vitamin D.

\section{Subjects and Methods:}

\section{Subjects:}

Patients were recruited from outpatient clinic of Hepatology, Gastroenterology and Infectious diseases in Benha university Hospital from October 2019 to October 2020. All patients were screened using a questionnaire on their medical history, symptoms, and findings in physical examination

\section{Inclusion criteria:}

1. Both sexes were included

2. Patients with non alcoholic fatty liver sonographically proven

3. All patients were $>18$ years of age

\section{Exclusion criteria:}

1. Evidence of other causes of chronic liver disease, including hepatitis B, C virus and autoimmune hepatitis

2. Presence of malignancies, ascites

3. The use of medications (current or within the last 12 month) known to induce hepatic steatosis (estrogens, corticosteroids, amiodarone, valproate
4. Inflammatory bowel disease and human immunodeficiency virus (HIV) infections

5. History of alcohol consumption

\section{Methods:}

A total of 95 patients were enrolled in this cross-sectional study and divided into 2 groups according to their ultrasound: 45 patients with sonographically evidenced non alcoholic fatty liver disease and 50 subjects with no sonographically evidenced non alcoholic fatty liver disease. An informed written consent was collected before study. The whole protocol was approved by the Ethics Committee of Benha faculty of medicine for approval. Weight was measured with a plethysmography scale, where patients wore minimal clothing, and height was measured with a stadiometer. Body mass index (BMI) was calculated by dividing the weight by height squared $(\mathrm{kg} / \mathrm{m} 2)$. Waist circumference (WC) was measured horizontally at the level of anterior superior iliac spine.

After overnight fasting, serum was collected. Biochemical markers, including Total cholesterol (TC), Triglyceride (TG), High density lipoprotein (HDL), Low density lipoprotein (LDL), Fasting blood glucose (FBG), HbA1c, Alanine aminotransferase (ALT), Aspartate aminotransferase (AST), 
HBsAg, HCV Ab and were measured with standard biochemical methods. Serum level of active form of Vitamin D was done using ELISAtechnique.

NAFLD was diagnosed based on the ultrasonographic evidence of hepatic steatosis, such as Increase hepatic brightness: defined as a homogenously increased echogenicity or hyperecchogenicity, posterior attenuation of the right lobe, the increased contrast between right kidney and the liver, the loss of visualization of the right diaphragm and the diminished visibility of the intrahepatic vessels [13]. Liver ultrasonography was evaluated with single viewer operator in supine position.

\section{Statistical analysis:}

Statistical presentation and analysis of the results were done using SPSS computer package, (SPSS, version 21) for data management, (I) Descriptive statistics was presented

as: (1)Mean $(X) \pm$ Standard Deviation (SD) for quantitative data and (2) Frequency and percenatage for qualitative data (II) Analyticaal statistics:In the statistical comparison between the different groups, the significance of difference was tested using one of the following tests: ANOVA test (F value), Chi-square test (X2 value) and unpaired student t-test (two sided). P value> 0.05 was considered statistically non significant $\mathrm{P}$ value $\leq 0.05$ was considered statistically significant $\mathrm{P}$ value III) Correlation analysis (Pearson's test): used to examine relationship between variables

\section{Results:}

At baseline, the age, sex and height were comparable between the control group and the NAFLD group. Weight, Waist circumference, Body mass index, Diabetes mellitus, Hypertension and Cardiovascular disease were significantly higher in patients with NAFLD than those in the control group as shown in table (Table 1)

Patients with NAFLD had a significantly lower serum level of Vitamin D than patients without NAFLD as shown in (Table 2)

Also serum level of Vitamin D was significantly lower in patients with severe steatosis than those with mild and moderate steatosis as shown in (Table3) and (Figure1) Non parametric spearman's was used to analyze the correlation between Serum Vitamin D and NAFLD. Serum vitamin D played a very important role in the process of the influence on NAFLD. Significant negative correlation was found between 
grades of steatosis detected by ultrasonography with serum level of Vitamin D measured by ELISA as presented in (Table 4) and (figure 2)

Significant positive correlation between grades of steatosis and laboratory findings was found regarding (Alanine aminotransferase, Aspartate aminotransferase, Fasting blood glucose, HbA1c, Serum cholesterol and
Triglycerides). but there was no significant correlation regarding Low density lipoprotein, High density lipoprotein as shown in

(Table

5)

There was significant negative correlation between serum level of Vitamin $D$ and (Alanine aminotransferase, Aspartate aminotransferase, HbA1c, Serum cholesterol and Triglycerides) as shown in (Table 6)

Table (1): Basic characteristcs of the studied groups

\begin{tabular}{|c|c|c|c|c|}
\hline & NAFLD $(n=45)$ & Non NAFLD $(\mathbf{n}=50)$ & $P$ value & Statistical Test \\
\hline$\overline{\text { Age }}$ & $41.09 \pm 10.33$ & $38.22 \pm 12.05$ & 0.218 & St t \\
\hline \multicolumn{5}{|l|}{ Sex } \\
\hline - Male & $21(46.7 \%)$ & $28(56 \%)$ & 0.414 & X2 test \\
\hline - Female & $24(53.3 \%)$ & $22(44 \%)$ & & \\
\hline \multicolumn{5}{|l|}{ Residence } \\
\hline - Rural & $27(60 \%)$ & $22(44 \%)$ & 0.119 & $\mathrm{X} 2$ test \\
\hline - Urban & $18(40 \%)$ & $28(56 \%)$ & & \\
\hline \multicolumn{5}{|l|}{ Occupation } \\
\hline - Farmer & $26(58 \%)$ & $16(36 \%)$ & $0.012 *$ & $\mathrm{X} 2$ test \\
\hline - Not farmer & $19(42 \%)$ & $34(64 \%)$ & & \\
\hline \multicolumn{5}{|l|}{ Marital status } \\
\hline - $\quad$ Married & $27(60 \%)$ & $14(28 \%)$ & 0.217 & $\mathrm{X} 2$ test \\
\hline - Not married & $18(40 \%)$ & $36(72 \%)$ & & \\
\hline Weight $(\mathrm{Kg})$ & $91.60+12.43$ & $67.16+8.29$ & $0.001 *$ & T test \\
\hline Height (cm) & $162.56 \pm 8.44$ & $163.44 \pm 9.52$ & 0.634 & T test \\
\hline BMI & $34.62+3.84$ & $24.59+3.13$ & $0.000 *$ & T test \\
\hline WC (cm) & $102.56+8.80$ & $82.64+6.68$ & $0.002 *$ & T test \\
\hline Smoking & & & & $\mathrm{X} 2$ test \\
\hline - Non smoker & $32(71.1 \%)$ & $37(74 \%)$ & 0.936 & \\
\hline - Smoker & $13(28.9 \%)$ & $13(26 \%)$ & & \\
\hline \multicolumn{5}{|l|}{ History of: } \\
\hline DM & $23(51.1 \%)$ & $6(12 \%)$ & $0.001 *$ & \\
\hline HTN & $21(46.7 \%)$ & $9(18 \%)$ & $0.003 *$ & \\
\hline CVD & $8(18.2 \%)$ & $1(2 \%)$ & $0.009 *$ & \\
\hline COPD & $6(13.3 \%)$ & $2(4 \%)$ & 0.102 & \\
\hline
\end{tabular}

BMI (Body mass index), WC (Waist circumference), DM (Diabetes mellitus), HTN (Hypertension), CVD (Cardiovascular disease), COPD (Chronic obstructive pulmonary disease), NAFLD (non alcoholic fatty liver disease), X2 test (chi square test), St t (Student's t -test), 
Table (2): Comparison between both groups regarding serum level of Vitamin D

\begin{tabular}{|c|c|c|c|c|}
\hline Serum Vitamin D $(20-70 \mathrm{ng} / \mathrm{ml})$ & $\begin{array}{c}\text { NAFLD } \\
(\mathbf{n = 4 5 )} \\
18.68+8.73\end{array}$ & $\begin{array}{c}\text { Non NAFLD } \\
(\mathbf{n = 5 0 )} \\
30.99+13.23\end{array}$ & $\begin{array}{c}\text { P value } \\
0.000 *\end{array}$ & $\begin{array}{c}\text { Statistical } \\
\text { Test } \\
\text { T test }\end{array}$ \\
\hline
\end{tabular}

NAFLD (non alcoholic fatty liver disease), St t (Student's t -test)

Table (3): Comparison between different grades of NAFLD regarding serum level of Vitamin D

\begin{tabular}{|c|c|c|c|c|c|c|}
\hline & \multicolumn{3}{|c|}{ NAFLD $($ Mean \pm S.D $)$} & \multirow{2}{*}{$\begin{array}{c}\text { Non } \\
\text { NAFLD } \\
(\mathbf{N}=50) \\
(\text { Mean+S.D) }\end{array}$} & \multicolumn{2}{|c|}{ Statistical test } \\
\hline & $\begin{array}{l}\text { Mild } \\
(\mathbf{N}=17)\end{array}$ & $\begin{array}{l}\text { Moderate } \\
(\mathbf{N}=\mathbf{2 3})\end{array}$ & $\begin{array}{l}\text { Severe } \\
(\mathbf{N}=5)\end{array}$ & & $\begin{array}{r}P \\
\text { value }\end{array}$ & $\operatorname{ANOVA}(\mathbf{F})$ \\
\hline Serum vitamin $D(20-70 \mathrm{ng} / \mathrm{ml})$ & $21.04 \pm 7.69$ & $18.26 \pm 9.54$ & $12.56+5.42$ & $\begin{array}{c}30 . \overline{9} 9 \pm 13 . \\
23\end{array}$ & $\begin{array}{l}0.002 \\
*\end{array}$ & 10.10 \\
\hline
\end{tabular}

S.D (standard deviation), NAFLD (non alcoholic fatty liver disease)

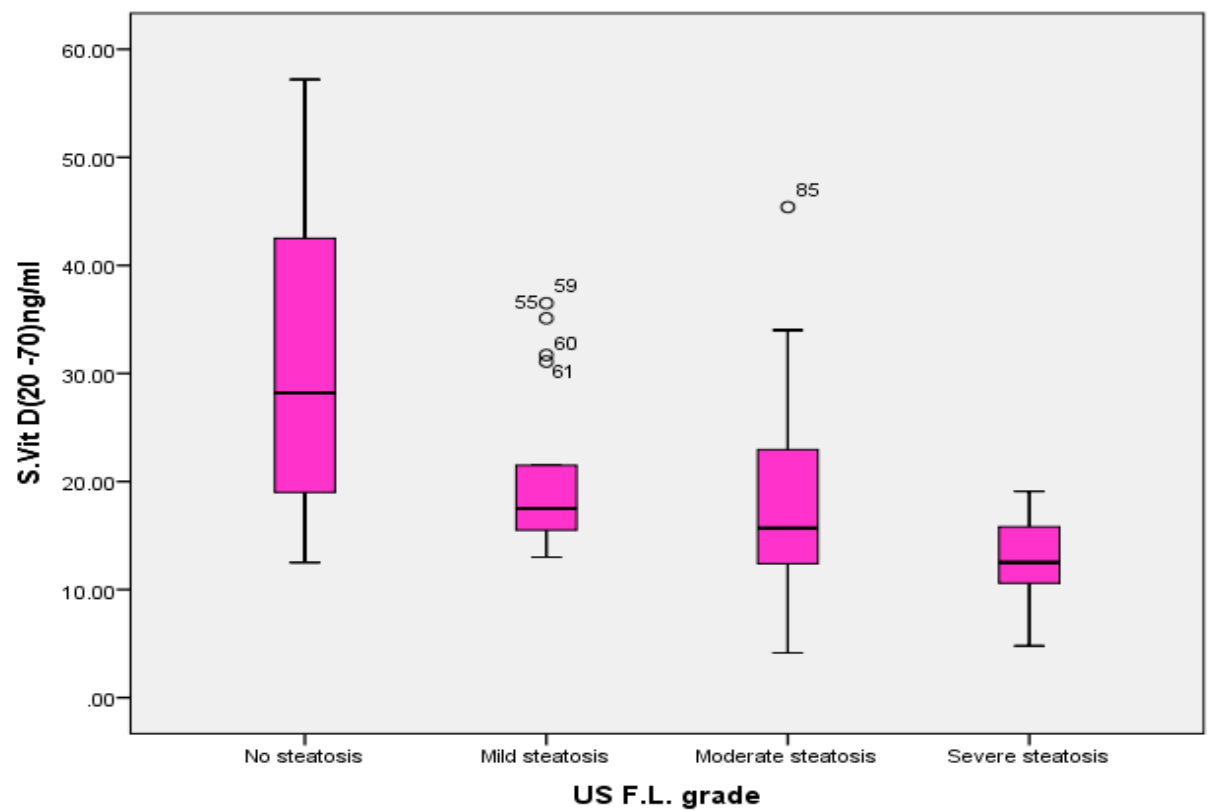

Fig.(1): Bar Chart showing comparison between different grades of NAFLD and serum level of Vitamin D

Table (4): Non parametric spearman's correlation between the grades of steatosis and serum level of Vitamin D

\begin{tabular}{ccc}
\hline & & US F.L. grade \\
Serum Vitamin D $(\mathbf{2 0 - 7 0} \mathbf{~ n g / m l})$ & R & -0.51 \\
\hline
\end{tabular}

F.L (fatty liver ), US (Ultrasound) 


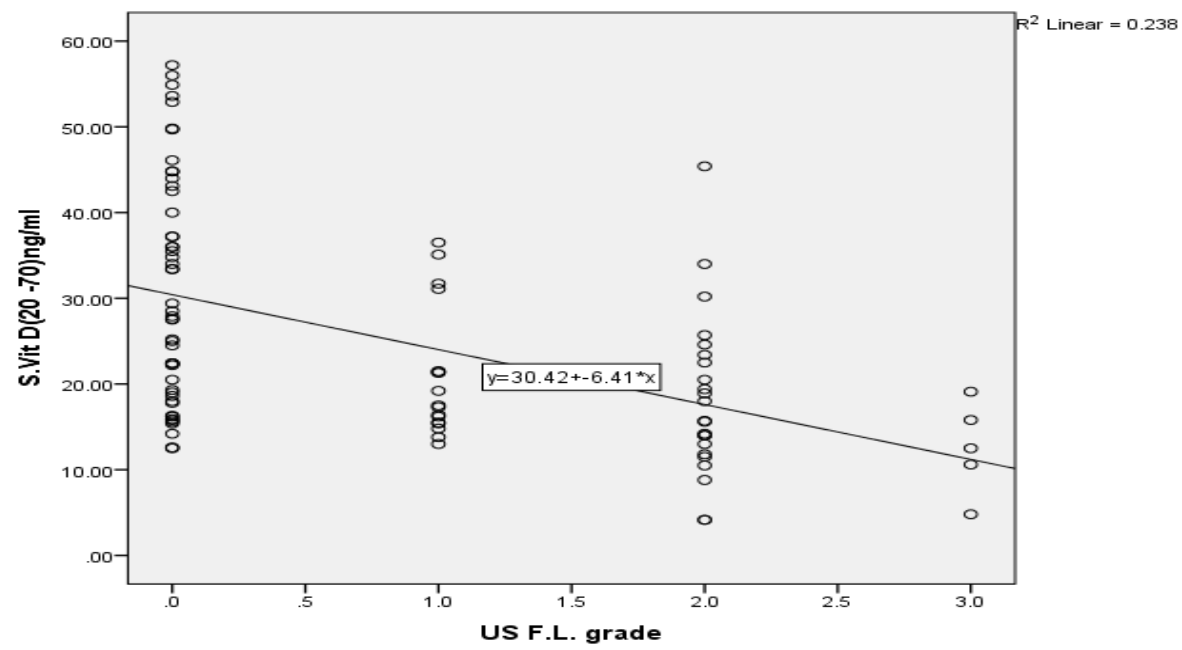

Fig. (2): Scatterplot showing negative correlation between grades of steatosis and serum level of Vitamin D

Table (5): Non parametric spearman's correlation between grades of steatosis and laboratory findings

\begin{tabular}{|c|c|c|}
\hline & & US F.L. grade \\
\hline \multirow[t]{2}{*}{ ALT } & $\mathbf{R}$ & 0.682 \\
\hline & $\mathbf{P}$ value & $<0.001^{*}$ \\
\hline \multirow[t]{2}{*}{$\mathbf{A S T}$} & $\mathbf{R}$ & 0.623 \\
\hline & $P$ value & $<0.001^{*}$ \\
\hline \multirow[t]{2}{*}{ FBG } & $\mathbf{R}$ & 0.390 \\
\hline & $\mathbf{P}$ value & $<0.001 *$ \\
\hline \multirow[t]{2}{*}{ HbA1C } & $\mathbf{R}$ & 0.498 \\
\hline & $\mathbf{P}$ value & $<0.001^{*}$ \\
\hline \multirow[t]{2}{*}{ Serum cholesterol } & $\mathbf{R}$ & 0.665 \\
\hline & $\mathbf{P}$ value & $<0.001^{*}$ \\
\hline \multirow[t]{2}{*}{ TGs } & $\mathbf{R}$ & 0.572 \\
\hline & $\mathbf{P}$ value & $<0.001^{*}$ \\
\hline \multirow[t]{2}{*}{ LDL } & $\mathbf{R}$ & 0.045 \\
\hline & $P$ value & 0.665 \\
\hline \multirow[t]{2}{*}{ HDL } & $\mathbf{R}$ & -0.151 \\
\hline & $P$ value & 0.144 \\
\hline
\end{tabular}

ALT (Alanine aminotransferase), AST (Aspartate aminotransferase), FBG (Fasting blood glucose), TGs (Triglycerides), LDL (Low density lipoproteins), HDL (High density lipoproteins) F.L (fatty liver ), US (Ultrasound) 
Table (6): Correlation between serum level of Vitamin D and laboratory findings

\begin{tabular}{llll}
\hline & & S.Vit D & Statistical test \\
\hline ALT & $\mathbf{R}$ & -0.331 \\
AST & P value & $0.001^{*}$ & \\
& $\mathbf{R}$ & -0.338 & \\
FBG & $\mathbf{P}$ value & $0.001^{*}$ & Non parametric Spearman's \\
& $\mathbf{R}$ & -0.195 & \\
HbA1C & $\mathbf{P}$ value & 0.059 & \\
\multirow{2}{*}{ Serum cholesterol } & $\mathbf{R}$ & -0.278 \\
& $\mathbf{P}$ value & $0.006^{*}$ \\
TGs & $\mathbf{R}$ & -0.328 \\
& $\mathbf{P}$ value & $0.001^{*}$ \\
LDL & $\mathbf{R}$ & -0.297 \\
& $\mathbf{P}$ value & $0.003^{*}$ & \\
HDL & $\mathbf{R}$ & -0.186 & \\
& $\mathbf{P}$ value & 0.071 & \\
\hline
\end{tabular}

ALT (Alanine aminotransferase), AST (Aspartate aminotransferase), FBG (Fasting blood glucose), TGs (Triglycerides), LDL (Low density lipoproteins), HDL (High density lipoproteins)

\section{Discussion:}

Nonalcoholic fatty liver disease (NAFLD) is a pathological condition that encompasses a wide spectrum of liver damage, ranging from simple steatosis to nonalcoholic steatohepatitis (NASH), cirrhosis, and hepatocellular carcinoma. The pathogenesis of NAFLD is multifactorial and is characterized by overweight and insulin resistance (IR) [14]. Lipid accumulation and free fatty acid (FFA) distribution from adipose tissue to liver plays an important role in the pathogenesis of NAFLD [15]. Lower levels of $25(\mathrm{OH}) \mathrm{D}$ in patients with NAFLD might contribute to the progression of
NAFLD however the exact mechanism of Vitamin D deficiency and NAFLD is not

fully indicated [16]. NAFLD and low serum Vitamin D are associated with obesity (high BMI) [17]. In our study we used ultrasonograghy for diagnosis and determination of grades of NAFLD, this agreed with a study which reported that ultrasound has good sensitivity $(85 \%)$ and specificity (95\%) compared with histology in identifying moderate and severe steatosis ${ }^{[18]}$. In the current study NAFLD was presented more frequently in females $(53.3 \%)$ than males $(46.7 \%)$. This was similar to a different study which proved higher prevalence of 
NAFLD in females [19]. In our study, mean weight was significantly higher in patients with NAFLD $(91.60 \pm 12.43)$ than those without NAFLD (67.16 \pm 8.29$)$. Also, it was documented that NAFLD is highly associated with several metabolic conditions like T2DM and obesity [20]. This came in agreement with some study reporting that obesity is associated with nonalcoholic fatty liver disease (NAFLD) [21]. On the other hand it was reported that NAFLD can also be observed in non-obese individuals and has its own metabolic characteristics, such as higher transaminase and insulin levels, less insulin sensitivity

[22].

In the current study, diabetes was noted in 23 patients $(51.1 \%)$ ) with NAFLD compared to $6(12 \%)$ in those without so there was a statistically significant positive association between diabetes mellitus and NAFLD This agreed with Leite et al. who reported that Prevalence of NAFLD among patients with type 2 diabetes has been estimated to be as high as $69 \%$ based on ultrasound diagnosis [23]. Again it was reported that nearly twothirds of people with obesity and T2DM and half the patients with hyperlipidaemia and hypertension have fatty liver on ultrasound [24].
In the present study although BMI was higher in NAFLD group $(34.62 \pm 3.84)$ than control group with mean (24.59 \pm 3.13$)$, with statistically significant difference also there was statistically significant incease in BMI with increasing grades of NAFLD ( $P$ value 0.001). This agrees with that study that demonstrated that higher BMI was observed in the NAFLD than those without NAFLD group [25]. In contrast to our study it was proved that NAFLD can occur among individuals who are not obese and have a normal body mass index [26].

In our study there was no significant difference between group of NAFLD and group without NAFLD regarding COPD but frequency of COPD was significantly higher in moderate and severe grades of NAFLD than mild grade. This agreed with others who stated that patients with COPD have increased visceral fat, a known inflammatory and lipolytic fat deposit. Thus, increase in free fatty acids accumulating in the liver might lead to NAFLD development in COPD [27]. In the present study the liver biochemical profile, ALT and AST were significantly higher in NAFLD patients with mean $\quad(39.64 \pm 8.62$ and $35.96 \pm 8.72$ respectively). Also ALT and AST levels increased significantly with increase in 
hepatic steatosis ( $\mathrm{P}$ value <0.001). Some researchers mentioned that NAFLD is the most common cause of a symptomatic elevation of transaminase levels [28].

In our study serum cholesterol and triglycerides were highr in patients with NAFLD than those without with mean (201.07 $\pm 24.58, \quad 153.98 \pm 18.40$ respectively) but there were no significant difference regarding LDL and HDL. Also seum cholesterol and TGs levels increased significantly with increase hepatic steatosis ( $\mathrm{P}$ value <0.001). This came in agreement with $\mathrm{Wu}$ et al. who reported that high serum triglyceride (TG) levels and low serum highdensity lipoprotein HDL) levels are also common in patients with NAFLD [29]. Also Santhoshakumari et al. showed that patients with NAFLD had higher TC, LDL, and TG, and lower HDL as compared to the control group [30]

Vitamin D receptors are present in several cell types and play an important role in both energy metabolism and bone metabolism, $25(\mathrm{OH}) \mathrm{D}$ is a major circulating form of vitamin $\mathrm{D}$ and constitutes the best clinical indicator of Vitamin D stores [31]. Our study showed that serum level of Vitamin D is lower in patients with NAFLD(18.68 \pm 8.73$)$ than those without $(30.99 \pm 13.23$ )and this level is much lower with increasing severity of steatosis with mean $(12.56 \pm 5.42)$ in case of severe steatosis in comparison to $(21.04 \pm 7.69)$ in mild steatosis with significant negative correlation between grades of FL and serum level of Vitamin D ( $\mathrm{P}$ value $<0.001$ ).Targher et al. found a close association between Vitamin D deficiency and NAFLD regarding histological severity of hepatic steatosis, necro-inflammation, and fibrosis [32]. Also Jiayancai et al. reported that the level of $25(\mathrm{OH}) \mathrm{D}$ in patients with mild, moderate and severe NAFLD was significantly lower in patients with NAFLD than those without NAFLD[33]

This study still had some limitations. Firstly, the small sample size which might limit the generalizability of the result. We could not perform hepatic biopsy to evaluate the severity of NAFLD because of invasiveness and Liver ultrasonography was considered an effective way to investigate NAFLD. Further studies are needed to evaluate the effect of Vitamin D supplementation on NAFLD in different extent. For example, we could conduct magnetic resonance spectroscopy (MRS) on the subjects or a hepatic biopsy

\section{Conclusion}

Patients with nonalcoholic fatty liver disease (NAFLD) had lower level of serum Vitamin $\mathrm{D}$ in comparison with patients without NAFLD and this significantly decreased with 
increasing with grades of fatty liver, also its

level was lower in patients with overweight

NAFLD than NAFLD patients with normal BMI

\section{References:}

1.Marjot, T; Moolla, A; Cobbold, JF and et al., (2020):

Nonalcoholic Fatty Liver Disease in Adults: Current

Concepts in Etiology, Outcomes, and Management".

Endocrine Reviews. 41 (1): bnz009.

2.Younossi ZM, Koenig AB, Abdelatif D and et al., (2016): Global epidemiology of nonalcoholic fatty liver disease-Meta-analytic assessment of prevalence, incidence, and outcomes. Hepatology. ;64:73-84.

3.Idilman IS, Keskin O, Celik A et al., (2016): Acomparison of liverfat conent asdetermined by magnetic resonance proton density fat fraction and MRS versus liver histology in non-alcoholic liver disease. Acta Radio;57:271-278.

4.Khanal U.P., Paudel B., Gurung G., and et al., (2019): Correlational study of nonalcoholic fatty liver disease diagnosed by ultrasonography with lipid profile and body mass index in adult Nepalese population. J. Med. Ultrasound.;27:19-25.

5.Bohte AE, Van Werven GR, Bipat S et al., (2011):The diagnostic accuracy of US,CT,MRI and H-MRS for the evaluation of hepatic steatosis compared with liver biopsy :a metaa-analysis.EurRadiol;21:87-97.

6.Vajro P, Maddaluno S and Veropalumbo C (2013): Persistent hypertransaminasemia in asymptomatic children: a stepwise approach. World J Gastroenterol.;19:2740-2751.

7.Zhou QG, Hou FF, Guo ZJ et al., (2004): 1,25Dihydroxyvitamin D improved the free fatty-acidinduced insulin resistance in cultured $\mathrm{C} 2 \mathrm{C} 12$ cells. Diabetes Metab Res Rev., 24: 459-464.
8.Wang X, Li W, Zhang Y and et al., (2015): Association between Vitamin D and non-alcoholic fatty liver disease/non-alcoholic steatohepatitis: Results from a meta-analysis. Int J Clin Exp Med.;8:17221-34.

9.Zhou QG, Hou FF, Guo ZJ et al., (2004): 1,25Dihydroxyvitamin D improved the free fatty-acidinduced insulin resistance in cultured $\mathrm{C} 2 \mathrm{C} 12$ cells. Diabetes Metab. Res. Rev.; 24: 459-64.

10.Wang X, Li W, Zhang Y et al., (2015): Association between vitamin $\mathrm{D}$ and non-alcoholic fatty liver disease/non-alcoholic steatohepatitis: Results from a meta-analysis. Int $\mathrm{J}$ Clin Exp Med.;8:1722117234.

11.Kwok RM, Torres DM and Harrison SA (2013): Vitamin D and nonalcoholic fatty liver disease (NAFLD): is it more than just an association?Hepatology.;58:1166-74.

12.Abramovitch S., Sharvit E., Weisman Y., et al., (2015): Vitamin D inhibits development of liver fibrosis in an animal model but cannot ameliorate established cirrhosis. Am. J. Physiol. Gastrointest. Liver Physiol.;308:G112-G120.

13.Van Werven J.R. , Marsman H. A., Nederveen A. J. and et al., (2010): Assessment of hepatic steatosis in patients undergoing liver resection: Comparison of US, CT, T1-weighted dual-echo MR imaging, and point-resolved 1H MR spectroscopy," Radiology, vol. 256 ,

no.

1 ,

pp. 159168.

14.Moon S.-S. , Y.-S. Lee, and S. W. Kim (2012): Association of nonalcoholic fatty liver disease with low bone mass in postmenopausal women," Endocrine, vol. 42, no. 2, pp. 423-429. 
15.Feng R, Luo C, Li C et al., (2017): Free fattyacids profile among lean, overweight and obese nonalcoholicfatty liver disease patients: a case - control study. Lipids HealthDis ;16:165.

16.Wang $\mathrm{X}$, Li W, Zhang $\mathrm{Y}$ and et al., (2015): Association between vitamin $\mathrm{D}$ and non-alcoholic fatty liver disease/non-alcoholic steatohepatitis: Results from a meta-analysis. Int $\mathbf{J}$ Clin Exp Med.;8:17221-17234.

17.Black LJ, Jacoby P, She Ping et al., (2014): low serum 25- hydroxyvitamin D concentrations associatewith non- alcoholic fatty liver disease in adolescents independent of adiposity.J Gastroenterol Hepatol 29:1215-12222.

18.Ballestri S, Nascimbeni F, Baldelli E and et al., (2017): Ultrasonographic fatty liver indicator detects mild steatosis and correlates with metabolic/histological parameters in various liver diseases. Metabolism ;72:57-65.

19.Rushad Patell, Rupal Dosi, Harshal Joshi, and et al., (2014): Non-Alcoholic Fatty Liver Disease (NAFLD) in Obesity. J Clin Diagn Res.; 8(1): 62-

66.

20.Ballestri, S., Nascimbeni, F., Romagnoli, D and et al., (2016): The independent predictors of nonalcoholic steatohepatitis and its individual histological features: Insulin resistance, serum uric acid, metabolic syndrome, alanine aminotransferase and serum total cholesterol are a clue to pathogenesis and candidate targets for treatment. Hepatol. Res. 46, 10741087.

21.Marchesini G, Bugianesi E, Forlani G and et al., (2003): Nonalcoholic fatty liver, steatohepatitis, and the metabolic syndrome. Hepatology.; 37:917923.
22. Vos B, Moreno C, Nagy N and et al., (2011): Lean non-alcoholic fatty liver disease (Lean-NAFLD): a major cause of cryptogenic liver disease. Acta Gastroenterol Belg.;74:389-

394.

23.Leite NC, Salles GF, Araujo ALE and et al., (2009):

Prevalence and associated factors of non-alcoholic fatty liver disease in patients with type-2 diabetes mellitus. Liver Int.;29:113-

119.

24.López-Suárez A, Guerrero JM, Elvira-González J and et al., (2011): Nonalcoholic fatty liver disease is associated with blood pressure in hypertensive and nonhypertensive individuals from the general population with normal levels of alanine aminotransferase Eur J Gastroenterol Hepatol.; 23(11):1011-7.

25.Greco EA, Francomano D, Fornari R and et al., (2013): Negative association between trunk fat, insulin resistance and skeleton in obese women. World J Diabetes 4(2):31-

39.

26.Chalasani N, Younossi Z, Lavine JE, et al., (2018): The diagnosis and management of nonalcoholic fatty liver disease: practice guidance from the American Association for the Study of Liver Diseases. Hepatology ;67:328357.

\section{Van den Borst B, Gosker HR and Schols AMWJ} (2013): Central fat and peripheral muscle: partners in crime in chronic obstructive pulmonary disease. Am. J. Respir. Crit. Care Med.;187:8-13.

28.Chalasani N, Younossi Z, Lavine JE and et al., (2012): The diagnosis and management of non-alcoholic fatty liver disease: Practice guideline by the American 
Benha medical journal, vol. 38, issue 2, 2021

Gastroenterological Association, American

Association for the Study of Liver Diseases, and American College of Gastroenterology.

Gastroenterology.;142:1592-609.

29.Wu KT, Kuo PL, Su SB and et al., (2016): Nonalcoholic fatty liver disease severity is associated with the ratios of total cholesterol and triglycerides to high-density lipoprotein cholesterol. J Clin Lipidol ;10:420-425.e1.

30.Santhoshakumari TMJ, Radhika G and Kanagavalli P (2017): A study of anthropometric and lipid profile parameters in non-alcoholic fatty liver disease patients attending a tertiary care hospital at puducherry. IOSR J Dent Med Sci (IOSR-JDMS) ;16:337.

To cite this article: Mustafa s. Elkady, Hatem S. Alegaily, Hany R. ElKholy, Shorouk Z. AbdElshafy, Amal A. Qayed. Serum Vitamin D in Patients with Non Alcoholic Fatty Liver Disease. BMFJ 2021;38(2):712-724. DOI: 10.21608/bmfj.2021.71838.1408 